\title{
Cost benefits of cardiovascular magnetic resonance imaging and angiography performed earlier in the diagnostic assessment of neonatal complex congenital heart disease
}

\author{
Pierangelo Renella ${ }^{1,2^{*}}$, Derek Phan ${ }^{3}$, Sarah N Khan ${ }^{1}$, Brian Reemtsen ${ }^{4}$, J Paul Finn ${ }^{1}$, Gary M Satou ${ }^{2}$ \\ From 17th Annual SCMR Scientific Sessions \\ New Orleans, LA, USA. 16-19 January 2014
}

\section{Background}

Neonates with complex congenital heart disease (CCHD) often require transcatheter or surgical intervention in the first month of life. Transthoracic 2D/Doppler echocardiography (2DE) is the diagnostic modality of choice, but occasionally may be inadequate for surgical planning. Unresolved pre-operative questions may require complete delineation of extracardiac vascular anatomy and/or ventricular volumes. In these cases, cardiac magnetic resonance imaging and angiography (CMR/MRA) may be delayed in favor of repeat 2DE studies, which often remain inconclusive. This approach may lead to unnecessary costs and has yet to be formally evaluated.

\section{Methods}

A retrospective review of neonates with CCHD who underwent CMR/MRA (2007-12) was performed to compare the costs of imaging between two groups of patients: one undergoing CMR/MRA after the initial 2DE but prior to any additional 2DE studies (Group A), and those with multiple 2DE studies prior to CMR/MRA (Group B). Thirty-one patients were identified (median age at CMR/ MRA was 4 days; range 2-25 days). Five patients were excluded (three without CHD; two without 2DE prior to CMR/MRA). The number of additional 2DE studies (after the initial 2DE) performed prior to CMR/MRA in Group $\mathrm{B}$ as compared to Group A ranged from one to three. A single CMR/MRA study prior to surgical intervention was obtained in all patients. All CMR/MRA studies were

'Radiology, David Geffen School of Medicine at UCLA, Los Angeles, California, USA

Full list of author information is available at the end of the article performed under general anesthesia (provided by a neonatologist) without adverse events.

\section{Results}

The total cost of cardiac imaging (2DE and CMR/MRA) in Group A $(\mathrm{n}=12)$ was $\$ 26,896(2 \mathrm{DE}=\$ 14,603$; CMR/ MRA $=\$ 12,293)$. The total cost in Group B $(\mathrm{n}=14)$ was $\$ 50,495(2 \mathrm{DE}=\$ 36,153 ; \mathrm{CMR} / \mathrm{MRA}=\$ 14,342)$. In total, $\$ 23,599$ less was spent in Group A as compared to Group $B$, with a mean per patient cost savings of $\$ 1,366$ between the two imaging strategies.

\section{Conclusions}

CMR/MRA has been shown to be of incremental benefit in the preoperative assessment of CCHD. However, depending on practice patterns and/or local availability of CMR, a diagnostic approach using multiple/serial 2DE studies is often utilized. This study is the first to suggest that a strategy involving the earlier use of CMR/

\section{Table 1 Patient Diagnostic Categories}

\begin{tabular}{cc}
\hline & $\begin{array}{c}\text { Number of Patients }(\mathbf{n}= \\
\text { 26) }\end{array}$ \\
\hline Anomalous Pulmonary Venous & 1 \\
Connection & 4 \\
Coarctation of the Aorta & 10 \\
Conotruncal Defects & 1 \\
Double Inlet Left Ventricle & 2 \\
Heterotaxy syndrome & 2 \\
Tricuspid Atresia & 4 \\
Multiple Left-Sided Obstructive Lesions & 2 \\
Vascular Ring &
\end{tabular}




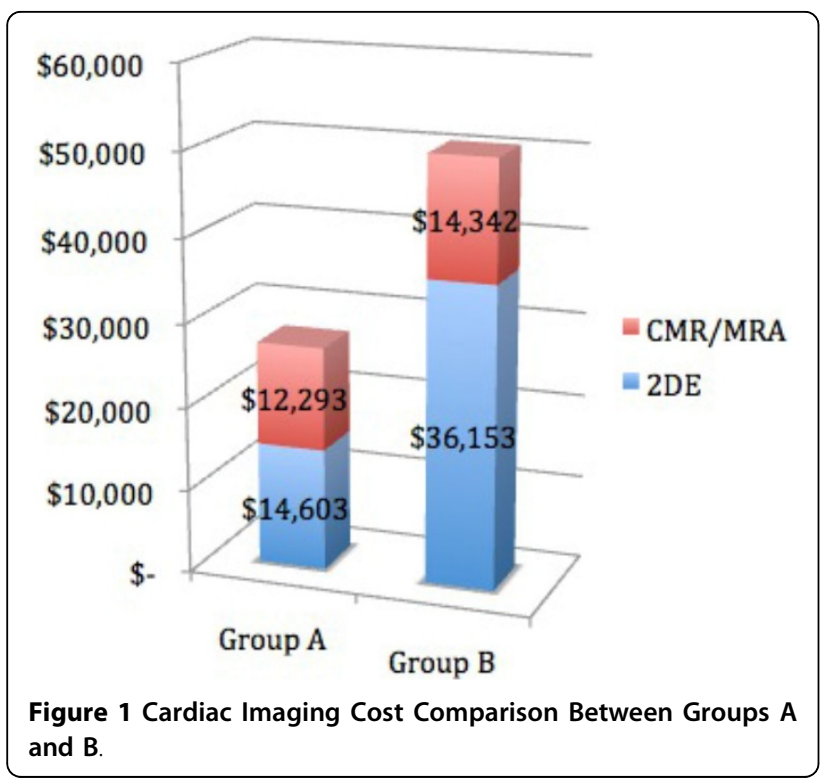

MRA in the diagnostic algorithm of CCHD patients may improve cost efficiency and cost savings. Further investigation that includes quantification of the diagnostic power of both 2DE and CMR/MRA across several congenital heart disease states may better define whether earlier and more routine use of CMR/MRA is cost effective.

\section{Funding}

None.

\section{Authors' details}

'Radiology, David Geffen School of Medicine at UCLA, Los Angeles, California, USA. ${ }^{2}$ Pediatrics, David Geffen School of Medicine at UCLA, Los Angeles, California, USA. ${ }^{3}$ David Geffen School of Medicine at UCLA, Los Angeles, California, USA. ${ }^{4}$ Pediatric Cardiothoracic Surgery, David Geffen School of Medicine at UCLA, Los Angeles, California, USA.

Published: 16 January 2014 